JOURNAL OF

SYMPLECTIC GEOMETRY

Volume 2, Number 3, 377-392, 2004

\title{
GENERIC BEHAVIOR OF ASYMPTOTICALLY HOLOMORPHIC LEFSCHETZ PENCILS
}

\author{
Jaume Amorós, Vicente Muñoz, and Francisco Presas
}

We prove that the vanishing spheres of the Lefschetz pencils constructed by Donaldson on symplectic manifolds of any dimension are conjugated under the action of the symplectomorphism group of the fiber. More precisely, a number of generalized Dehn twists may be used to conjugate those spheres. This implies the non-existence of homologically trivial vanishing spheres in these pencils. To develop the proof, we discuss some basic topological properties of the space of asymptotically holomorphic transverse sections.

\section{Introduction}

In this article we analyze the generic behavior of vanishing spheres in the symplectic pencils introduced by Donaldson in [10], henceforth referred to as Donaldson's $\varepsilon$-transverse Lefschetz pencils (see Section 2 for precise definitions), and show it to be similar to the case of Lefschetz pencils for complex projective varieties. Using the pencils as a tool, we start the study of the symplectic analogue of the dual variety in algebraic geometry, which we believe will be of interest in symplectic topology.

The property of Lefschetz pencils on projective algebraic varieties that we seek to extend is classical:

Theorem 1.1 (cf. [18] XVIII, 6.6.2). Let $M$ be a complex projective manifold, and $L \rightarrow M$ an ample line bundle. For $k$ large enough the pencils associated to quotients of holomorphic sections of the line bundle $L^{\otimes k}$ have vanishing spheres which are conjugated under the action of the group $\operatorname{Diff}^{+}(F)$ of orientation-preserving diffeomorphisms of the generic fiber $F$. 
The main result of this article is a generalization of the previous theorem to the symplectic case. By establishing the irreducibility of symplectic analogues to the dual variety, defined in (1), we will prove a more precise version, which replaces the diffeomorphism group of $F$ with its smallest possible subgroup. For that we need the following

Definition 1.2. Two Lagrangian submanifolds $L_{0}$ and $L_{1}$ are Lagrangian isotopic if there is a continuous family of Lagrangian submanifolds $\left\{L_{t}\right\}_{t \in[0,1]}$ connecting them.

Then, we show

Theorem 1.3. For $k$ large enough Donaldson's $\varepsilon$-transverse Lefschetz pencils have vanishing spheres that, up to Lagrangian isotopy, are conjugated under the action of the group of symplectomorphisms generated by the Dehn twists of the fiber along the vanishing spheres of the pencil itself.

We point out that the conjugating group of symplectomorphisms of Theorem 1.3 is not elementary, as Donaldson's pencils exist for large $k$ and have $O\left(k^{\operatorname{dim} M / 2}\right)$ singular fibers. Nevertheless, this group may be substantially smaller than the full symplectomorphism group of the fiber. Note that its action on the homology of the fiber is trivial except in middle degree, while in many instances, such as products and bundles of symplectic manifolds, there exist symplectomorphisms acting nontrivially in other cohomology groups.

Finally we will give the following application of theorem 1.3:

Theorem 1.4. For $k$ large enough Donaldson's $\varepsilon$-transverse Lefschetz pencils satisfy that all the vanishing spheres are homologically non-trivial.

In the case of dimension 4, I. Smith [16] has already proved that the vanishing spheres appearing in the Donaldson's $\varepsilon$-transverse Lefschetz pencils are homologically non-trivial for $k$ even. On the other hand, for general symplectic Lefschetz pencils in 4-manifolds [8] gives a lower bound for the number of homologically non-trivial vanishing spheres.

In the cases of dimension greater than 4 , Theorem 1.4 is still a meaningful result, as homologically trivial Lagrangian spheres are known to exist in higher dimensional symplectic manifolds at least in dimension $4 k+2$ (see the examples of $[\mathbf{1 7}, 3 . \mathrm{d}]$ and $[\mathbf{2}]$, for instance), and there is great flexibility in constructing symplectic Lefschetz pencils adapted to given Lagrangian submanifolds $([\mathbf{7}])$.

Theorem 1.1 is a consequence of the irreducibility of the dual variety, which is the subset of the dual projective space defined by hyperplanes tangent to an embedded projective variety. It is not too hard to give a definition of this variety in the symplectic case, by combining ideas of [14] and [10]. This dual "symplectic" variety appears as an "asymptotically holomorphic" divisor. The divisor is not smooth and, in particular, has self-intersections. 
It is difficult to control the behavior of self-intersections for this kind of divisor (for an example without known solution see [5]). Therefore we have chosen an alternative route to prove Theorem 1.3. However it makes sense to speak in a "rough sense" of the complement of the dual variety, i.e., the space of transverse hyperplanes. We will give some topological properties of this space.

The organization of this paper is as follows. In Section 2 we give the definitions and results introduced in $[\mathbf{9}, \mathbf{1 0}]$ needed to carry out the proofs. In Section 3 we establish our symplectic analogue to the irreducibility of the dual variety, on which the whole paper rests. A discussion of the topology of the set of "transverse" sections is carried out in Section 4. Using this, Theorems 1.3 and 1.4 are proved in Section 5. Finally, in Section 6 we briefly discuss the possibility of obtaining a symplectic invariant from the space of transverse sections.

Acknowledments. Some of the results contained in Section 4 are the result of conversations between D. Auroux and the third author. In particular, the statement of Theorem 4.2 was suggested by him to us. We thank Universidad Autónoma de Madrid for its hospitality to the first author during the elaboration of this work. We are also grateful to L. Katzarkov and I. Smith for useful comments.

\section{Donaldson's asymptotically holomorphic theory}

Let $(M, \omega)$ be a symplectic manifold of integer class, i.e., satisfying that $[\omega] / 2 \pi$ admits an integer lift to $H^{2}(M ; \mathbb{Z})$. We define $L$ to be a hermitian bundle with connection whose curvature is $R_{L}=-i \omega$. Moreover fixing a compatible almost complex structure $J$, this defines a Riemannian metric $g(u, v)=\omega(u, J v)$ on $M$. We also consider the sequences of metrics $g_{k}=k g$, $k \geq 1$. The following definitions are needed.

Definition 2.1. A sequence of sections $s_{k}$ of the bundles $L^{\otimes k}$ has $C^{3}$-bounds $c$ if it satisfies the following bounds (in $g_{k}$-metric)

$$
\left|s_{k}\right| \leq c, \quad\left|\nabla^{r} s_{k}\right| \leq c \quad(r=1,2,3), \quad\left|\nabla^{r} \bar{\partial} s_{k}\right| \leq c k^{-1 / 2} \quad(r=1,2) .
$$

Definition 2.2. A sequence of sections $s_{k}$ of the hermitian bundles $E_{k}$ is $\varepsilon$-transverse to zero at the point $x \in M$ if one the two following conditions is fulfilled

i) $\left|s_{k}(x)\right| \geq \varepsilon$.

ii) $\nabla s_{k}: T_{x} M \rightarrow\left(E_{k}\right)_{x}$ is surjective and it admits a right inverse of norm bounded by $\varepsilon^{-1}$, using the norm $g_{k}$ in $M$.

The sequence is said to be transverse to zero in a set $U$ if it is transverse to zero at every point of the set. 
We need to introduce notations to control 1-parametric families of sections. In particular $\left(J_{t}\right)_{t \in[0,1]}$ will denote a 1-parametric family of almost complex structures compatible with $\omega$. We can associate to this family a sequence of families of metrics $g_{k, t}$. A family of sequences of sections $s_{k, t}$ has $C^{3}$-bounds $c$ if $s_{k, t}$ has $C^{3}$-bounds $c$ with respect to the almost complex structure $J_{t}$ and the metric $g_{k, t}$ for each $t \in[0,1]$. (Note that such a family is a continuous path in the space of sections of $L^{\otimes k}$ with the $C^{3}$-topology.) The same remark must be applied for families of $\varepsilon$-transverse sequences.

There exist local objects that are close to being holomorphic, as given by the following two lemmas

Lemma 2.3 (Lemma 3 in [4]). Let $x_{t} \in M, t \in[0,1]$, be a path in $M$. Then there exist complex Darboux coordinates depending continuously on $t, \Phi_{t}: B_{g_{k}}\left(x_{t}, k^{1 / 2}\right) \rightarrow B_{\mathbb{C}^{n}}\left(0, k^{1 / 2}\right), \Phi_{t}\left(x_{t}\right)=0$ such that the inverse $\Psi_{t}=\Phi_{t}^{-1}$ of the coordinate map is nearly pseudo-holomorphic with respect to the almost complex structure $J_{t}$ on $M$ and the canonical complex structure on $\mathbb{C}^{n}$. Namely, the map $\Psi_{t}$ satisfies $\left|\nabla^{r} \Psi_{t}\right|=O(1)$ for $r=1,2,3$ and $\left|\bar{\partial} \Psi_{t}(z)\right|=O\left(k^{-1 / 2}|z|\right)$, and $\left|\nabla^{r} \bar{\partial} \Psi_{t}\right|=O\left(k^{-1 / 2}\right)$ for $r=1,2$.

We also have

Lemma 2.4 (Lemma 3 in $[\mathbf{3}]$ ). There exist constants $\lambda>0$ and $c_{s}>0$ such that, given any continuous path $x_{t}:[0,1] \rightarrow M$, and large $k$, there exist sections $s_{k, x_{t}}^{r e f}$ of $L^{\otimes k}$ over $M$ with the following properties:

i) The sections $s_{k, x_{t}}^{\text {ref }}$ have $C^{3}$-bounds with respect to $J_{t}$, independent of $t$.

ii) They depend continuously on $t$.

iii) The bound $\left|s_{k, x_{t}}^{r e f}\right|>c_{s}$ holds over the ball of $g_{k}$-radius 1 around $x_{t}$.

iv) $\left|s_{k, x_{t}}^{r e f}(q)\right| \leq \exp \left(-\lambda d_{k}\left(x_{t}, q\right)^{2}\right)$, where $d_{k}$ is the distance associated to $g_{k}$.

v) $s_{k, x_{t}}^{r e f}$ is supported in a ball around $x_{t}$ of $g_{k}$-radius $c k^{1 / 6}$ for some constant $c$.

Recall from [10]:

Definition 2.5. A symplectic Lefschetz pencil on $(M, \omega)$ is a surjective map $\phi: M-N \rightarrow \mathbb{C P}^{1}$, with $N$ a codimension 4 symplectic submanifold, such that every point $p \in M$ has a complex-valued coordinate neighbourhood $\psi: U \subset M \rightarrow \mathbb{C}^{n}$ sending $p$ to $(0, \ldots, 0)$, the standard symplectic structure $\omega_{0}$ of $\mathbb{C}^{n}$ to $\omega$, and such that

i) For $p \in N, N$ has local equation $\left\{z_{1}=z_{2}=0\right\}$, and $\phi\left(z_{1}, \ldots, z_{n}\right)=$ $z_{2} / z_{1}$.

ii) For finitely many critical points $p_{1}, \ldots, p_{\Lambda} \in M-N, \phi\left(z_{1}, \ldots, z_{n}\right)=$ $z_{1}^{2}+\cdots+z_{n}^{2}$ (the ordinary quadratic singularity in algebraic geometry).

iii) For all other points $p \in M-N, p \neq p_{1}, \ldots, p_{\Lambda}, \phi\left(z_{1}, \ldots, z_{n}\right)=z_{1}$. 
The main result of $[\mathbf{1 0}]$ is

Theorem 2.6 (Theorem 2 in $[\mathbf{1 0}]$ ). Given a symplectic manifold $(M, \omega)$ such that the cohomology class $[\omega] / 2 \pi$ has an integer lift to $H^{2}(M, \mathbb{Z})$, there exists a symplectic Lefschetz pencil whose fiber is homologous to the Poincaré dual of $k[\omega] / 2 \pi$, for $k$ large enough.

Donaldson's proof of Theorem 2.6 goes through the following two steps:

Proposition 2.7. Given a sequence of sections $s_{k}^{1} \oplus s_{k}^{2}$ of $L^{\otimes k} \oplus L^{\otimes k}$ which has $C^{3}$-bounds $c$, then for any $\delta>0$, there exists $\varepsilon>0$ and a sequence of sections $\sigma_{k}^{1} \oplus \sigma_{k}^{2}$ such that the following conditions are satisfied for $k$ large

i) $\left|s_{k}^{j}-\sigma_{k}^{j}\right|_{C^{1}} \leq \delta$, for $j=1,2$.

ii) $\sigma_{k}^{1}$ is $\varepsilon$-transverse to zero over $M$.

iii) $\sigma_{k}^{1} \oplus \sigma_{k}^{2}$ is $\varepsilon$-transverse to zero over $M$.

iv) Denoting by $Z_{k, \varepsilon}=\left\{p \in M:\left|\sigma_{k}^{1}\right| \leq \varepsilon\right\}$, the map $\partial\left(\sigma_{k}^{2} / \sigma_{k}^{1}\right)$ is $\varepsilon$ transverse to zero in $M-Z_{k, \varepsilon}$.

Moreover, given a 1-parametric family of sequences of sections $s_{k, t}^{1} \oplus s_{k, t}^{2}$, $t \in[0,1]$, of $L^{\otimes k} \bigoplus L^{\otimes k}$ which have $C^{3}$-bounds $c$, then there exists a family of sequences of sections $\sigma_{k, t}^{1} \oplus \sigma_{k, t}^{2}$ satisfying the properties above for each $t \in[0,1]$. The result also holds for continuous families of sequences of sections parametrized by $t \in S^{1}$.

The second step in the proof is

Proposition 2.8. Given $\rho>0$ and a sequence of sections $s_{k}^{1} \oplus s_{k}^{2}$ satisfying the last three properties of Proposition 2.7, then the maps

$$
\phi_{k}: M-Z\left(s_{k}^{1} \oplus s_{k}^{2}\right) \rightarrow \mathbb{C P}^{1}
$$

are well defined and for $k$ large enough there exists a perturbation producing a map $\hat{\phi}_{k}$ which defines a symplectic Lefschetz pencil and verifies $\| \hat{\phi}_{k}-$ $\phi_{k} \|_{C^{1}, g_{k}}<\rho$.

The same result holds for continuous families of sequences of sections $s_{k, t}^{1} \oplus s_{k, t}^{2}, t \in[0,1]$ or $t \in S^{1}$, satisfying the conditions of Proposition 2.7.

For proofs of these results we refer the reader to [10]. The 1-parametric version with parameter space $S^{1}$ follows easily from the version with parameter space $[0,1]$.

We will call Donaldson's $\varepsilon$-transverse Lefschetz pencils the Lefschetz pencils yielded by Proposition 2.8. The 1-parametric part of Proposition 2.8 shows that the symplectic Lefschetz pencils obtained by this procedure are all isotopic for any given $k$ large enough. 


\section{Vanishing spheres for symplectic Lefschetz pencils}

After blowing-up the symplectic submanifold $N$, any pencil becomes a fibration $\tilde{M} \rightarrow \mathbb{C P}^{1}$. We can define a canonical symplectic connection outside the critical points in this fibration using the symplectic orthogonal of the tangent space to the fiber as the horizontal subspace. The parallel transport and geometric monodromy so defined act by symplectomorphisms in the fibers (cf. [13, Lemma 6.18]).

It is possible to define a parallel transport ending in a singular fiber, and the local computation of the Picard-Lefschetz formula in algebraic manifolds (see [1, Vol. 2, Ch. 1]) shows that in this case Lagrangian spheres in the regular fibers contract to the singular point, forming a Lagrangian disk in the manifold $M$. These spheres are called the Lagrangian vanishing spheres in the regular fiber.

For a basepoint regular value $w \in \mathbb{C P}^{1}$, a Lagrangian vanishing sphere in the fiber $F=p^{-1}(w)$ is determined by every path from $w$ to a critical value $w_{i}$. The choice of a set of non-intersecting paths $\gamma_{1}, \ldots, \gamma_{s}$ from $w$ to the singular values $w_{1}, \ldots, w_{s}$ of the pencil defines a set of vanishing spheres in $F$ that, together with a choice of isotopy from the product of monodromies around all critical values to identity, determine the diffeomorphism type of the pencil, in particular of the total space $\tilde{M}([\mathbf{1 2}])$.

Lefschetz pencils on a projective manifold $M \hookrightarrow \mathbb{C P}^{N}$ are defined by lines in the dual projective space $\mathbb{C P}^{1} \cong l \subset\left(\mathbb{C P}^{N}\right)^{*}$ intersecting the dual variety $M^{*}$ transversely at smooth points. The hyperplane sections defined by the line $l$ cover $M$, are smooth outside the finite intersection $l \cap M^{*}$, where they consist of a hyperplane section with an ordinary quadratic singularity (see [18] XVII, XVIII for a complete discussion).

The conjugacy modulo diffeomorphism of Theorem 1.1 follows from the irreducibility of the dual variety $M^{*}$ for high $k$ : its smooth points form a connected open set $U \subset M^{*}$, and given two critical values $w_{i}, w_{j}$ of the pencil, a path connecting them in $U$ defines a smooth family of hyperplane sections of $M$ whose parallel transport induces the conjugacy of vanishing spheres.

In the symplectic case, instead of considering families of pencils interpreted as lines in the dual projective space, we consider the families in abstract. The result we want to prove is

Proposition 3.1. The sequences of pencils provided by Theorem 2.6 satisfy, for $k$ large enough, that all Lagrangian vanishing spheres of a generic fiber are equivalent under the action of an orientation-preserving diffeomorphism of the fiber.

Proof. Choose a sequence of sections $s_{k}=s_{k}^{1} \oplus s_{k}^{2}$ satisfying the conditions of Proposition 2.7, and let $\phi_{k}=s_{k}^{2} / s_{k}^{1}$ be the associated maps to $\mathbb{C P}^{1}$. 
For each $k$ select critical points $p_{0}$ and $p_{1}$ of the map $\partial \phi_{k}$. From [10] we know that these points will become the critical points of the pencil after the perturbation performed in Proposition 2.8. Now choose a path $p_{t}$ in $M$ joining $p_{0}$ and $p_{1}$. We can choose this path to avoid a $c^{\prime}$-neighborhood of $Z\left(s_{k}^{1}\right)$ for some uniform $c^{\prime}$, since by $[\mathbf{1 0}]$ the $\varepsilon$-transversality implies that $p_{0}$ and $p_{1}$ are away from a $c^{\prime}$-neighborhood of $Z\left(s_{k}^{1}\right)$, for large $k$. Also we may suppose that $p_{t}$ is stationary in $[0, \beta]$ and $[1-\beta, 1]$, for some small $\beta>0$. Also let $\lambda_{t}=\phi_{k}\left(p_{t}\right)$, which is a uniformly bounded path in $\mathbb{C}$.

Choose a family of sections $s_{k, p_{t}}^{\text {ref }}$ of $L^{\otimes k}$ satisfying the properties of Lemma 2.4 for the path $p_{t}$. We use these sections to trivialize $L^{\otimes k}$ in a neighborhood $U_{t}$ of fixed $g_{k}$-radius $O(1)$ of $p_{t}$. The map $\phi_{k}$ is given by

$$
\begin{aligned}
f_{k}: U_{t} & \rightarrow \mathbb{C} \\
q & \mapsto \frac{s_{k}^{2}}{s_{k}^{1}}(q),
\end{aligned}
$$

which has $C^{3}$-bounds $c_{1} c$ (with the obvious adapted definition) where $s_{k}$ has $C^{3}$-bounds $c$ and $c_{1}>0$ is a constant depending only on the geometry of the manifold (not depending on $k$, the initial sections, etc).

By Lemma 2.3, we may trivialize the neighborhood $U_{t}$ by a chart $\Phi_{t}$ : $B_{\mathbb{C}^{n}}(0,1) \rightarrow U_{t}, \Phi_{t}(0)=p_{t}$, and

$$
\hat{f}_{k, t}=f_{k} \circ \Phi_{t}: B_{\mathbb{C}^{n}}(0,1) \rightarrow \mathbb{C}
$$

has $C^{3}$-bounds $c_{2} c$ in the unit ball of $\mathbb{C}^{n}$. Moreover $\partial \hat{f}_{k, j}$ is $\varepsilon / c_{2}$-transverse to zero for $j=0,1$. Again the constant $c_{2}>0$ depends only on the geometry of the manifold $M$. Identifying the tangent space at the origin to $\mathbb{C}^{n}$ we define $\hat{h}_{k, j}, j=0,1$, as the quadratic form on $\mathbb{C}^{n}$ associated to $\partial \partial \hat{f}_{k, j}(0)$. Moreover, it is possible to construct a path of non-degenerate quadratic forms $\hat{h}_{k, t}$ starting and ending at the two previous quadratic forms. Also the eigenvalues of the quadratic forms of the path can be bounded below and above by the eigenvalues of the two initial quadratic forms. We can assume that the path $\hat{h}_{k, t}$ is stationary in $[0, \beta]$ and $[1-\beta, 1]$. Again $\partial \hat{h}_{k, t}$ is $\varepsilon / c_{3}$-transverse to zero and $\hat{h}_{k, t}$ has $C^{3}$-bounds $c_{3} c$ on $B_{\mathbb{C}^{n}}(0,1)$, where $c_{3}>0$ only depends on the geometry of the manifold. On the other hand, note that $\hat{h}_{k, t}$ is naturally defined all over $\mathbb{C}^{n}$. Now we define the following section to the trivialized bundle $\Phi_{t}^{*}\left(L^{\otimes k} \oplus L^{\otimes k}\right)$ on $B_{\mathbb{C}^{n}}(0,1)$ :

$$
\hat{l}_{k, t}=\left(1, \lambda_{t}+\hat{h}_{k, t}\right) \text {. }
$$

The goal now is to go back to the manifold. First, note that by Lemma 2.3 the chart $\Phi_{t}$ may be extended as

$$
\Phi_{t}: B_{\mathbb{C}^{n}}\left(0, k^{1 / 2}\right) \rightarrow V_{t}
$$


where $V_{t}$ is a neighborhood of $p_{t}$ of $g_{k}$-radius $O\left(k^{1 / 2}\right), p_{t} \in U_{t} \subset V_{t}$. Also

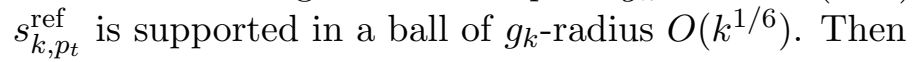

$$
u_{k, t}= \begin{cases}\frac{\beta-t}{\beta} s_{k}+\frac{t}{\beta}\left(\hat{l}_{k, 0} \circ \Phi_{0}^{-1}\right) \otimes s_{k, p_{0}}^{\mathrm{ref}}, & t \in[0, \beta] \\ \left(\hat{l}_{k, t} \circ \Phi_{t}^{-1}\right) \otimes s_{k, p_{t}}^{\mathrm{ref}}, & t \in[\beta, 1-\beta] \\ \frac{t-1+\beta}{\beta} s_{k}+\frac{1-t}{\beta}\left(\hat{l}_{k, 1} \circ \Phi_{1}^{-1}\right) \otimes s_{k, p_{1}}^{\mathrm{ref}}, & t \in[1-\beta, 1]\end{cases}
$$

is a well-defined section of $L^{\otimes k} \oplus L^{\otimes k}$. It is easy to check that $u_{k, t}$ has $C^{3}$ bounds $c_{4} c$, where $c_{4}$ depends only on the geometry of the manifold, using that $\left|\bar{\partial} \Phi_{t}(z)\right|=O\left(k^{-1 / 2}|z|\right)$ and that $s_{k, p_{t}}^{\text {ref }}$ has Gaussian decay. Now in a small neighborhood of $p_{t}$ the map

$$
\phi_{k, t}=\frac{u_{k, t}^{2}}{u_{k, t}^{1}}= \begin{cases}\frac{(1-\mu) s_{k}^{2}+\mu s_{k, p_{0}}^{\mathrm{ref}}\left(\lambda_{0}+\hat{h}_{k, 0} \circ \Phi_{0}^{-1}\right)}{(1-\mu) s_{k}^{1}+\mu s_{k, p_{0}}^{\mathrm{ref}}}, & \mu=\frac{t}{\beta} \in[0,1] \\ \lambda_{t}+\hat{h}_{k, t} \circ \Phi_{t}^{-1}, & t \in[\beta, 1-\beta] \\ \frac{(1-\mu) s_{k}^{2}+\mu s_{k, p_{1}}^{\mathrm{ref}}\left(\lambda_{1}+\hat{h}_{k, 1} \circ \Phi_{1}^{-1}\right)}{(1-\mu) s_{k}^{1}+\mu s_{k, p_{1}}^{\mathrm{ref}}}, & \mu=\frac{1-t}{\beta} \in[0,1]\end{cases}
$$

is well-defined, $\partial \phi_{k, t}$ has a zero in $p_{t}$ and is $\varepsilon / c_{4}$-transverse to zero in that small neighborhood. This is clear in the second interval. In the first and third intervals it is easy to check if we have previously normalized $s_{k, p_{t}}^{\text {ref }}$ to be a positive real multiple of $s_{k}^{1}$ at the point $p_{t}$. Note that $s_{k}^{1}$ is uniformly bounded below since we are working off a neighborhood of $Z\left(s_{k}^{1}\right)$.

Now we apply Proposition 2.7 to the family $u_{k, t}$ choosing $\delta=\min \left\{\frac{\varepsilon}{4 c_{4}}, \frac{\varepsilon}{2}\right\}$. We obtain a new family $\hat{u}_{k, t}$ which satisfies the required transversality conditions with a constant $\varepsilon^{\prime}$ depending on $\delta$. The map $\hat{\phi}_{k, t}=\frac{\hat{u}_{k, t}^{2}}{\hat{u}_{k, t}^{1}}$ is well defined outside $Z\left(\hat{u}_{k, t}\right)$. It is clear that there is a path of zeroes of $\partial \hat{\phi}_{k, t}$ which is $C^{0}$-close to $p_{t}$. Moreover, choosing $\alpha>0$ small, we define the family

$$
\tilde{s}_{k, t}= \begin{cases}\frac{\alpha-t}{\alpha} s_{k, 0}+\frac{t}{\alpha} \hat{u}_{k, 0}, & t \in[0, \alpha] \\ \hat{u}_{k, \frac{t-\alpha}{1-2 \alpha}} & t \in[\alpha, 1-\alpha] \\ \frac{t-1+\alpha}{\alpha} s_{k, 1}+\frac{1-t}{\alpha} \hat{u}_{k, 1}, & t \in[1-\alpha, 1]\end{cases}
$$

This defines a family of sections that are $\min \left(\varepsilon^{\prime}, \varepsilon\right)$-transverse to zero in the sense that they satisfy the various properties of Proposition 2.7. 
Therefore the points $p_{0}$ and $p_{1}$ are joined by a path of zeroes of $\partial \tilde{\phi}_{k, t}$, where $\tilde{\phi}_{k, t}$ is the map associated to $\tilde{s}_{k, t}$. After applying Proposition 2.8, this path becomes a path of critical points of the family of Lefschetz pencils. By the same arguments as in the algebraic case the vanishing spheres associated to the two critical points are conjugated through the action of an orientation-preserving diffeomorphism. The last point is to recall that the $C^{3}$-bounds and the estimated transversality constants obtained along the way are independent of the chosen points $p_{0}$ and $p_{1}$. This implies that the constructions begin to work for a given $k$ for all possible pairs of points. This concludes the proof.

Remark 3.2. The above argument may be continued to prove that the Lagrangian vanishing spheres are conjugated by symplectomorphisms of the fiber. To check this, it suffices to use the parallel transport defined by the symplectic orthogonal horizontal space in the family of Lefschetz pencils on $M$. We skip this refinement to prove directly the stronger statement of Theorem 1.3.

\section{Topology of the space of $\varepsilon$-transverse sections}

In the case of a complex projective manifold, where Lefschetz pencils arise from lines in the dual projective space, the embedding into it of the dual variety has a further property that facilitates the study of its complement:

Theorem 4.1 (Lefschetz hyperplane section theorem, Hamm-Lê, see [11]). Let $M \hookrightarrow \mathbb{C P}^{N}$ be a complex projective manifold, $M^{*} \hookrightarrow\left(\mathbb{C P}^{N}\right)^{*}$ its dual variety, and let $H \subset\left(\mathbb{C P}^{N}\right)^{*}$ be a generic linear subvariety of complex dimension d. Then the homotopy group morphisms $\pi_{i}\left(H-\left(H \cap M^{*}\right)\right) \rightarrow$ $\pi_{i}\left(\left(\mathbb{C P}^{N}\right)^{*}-M^{*}\right)$ induced by the natural inclusion of spaces are isomorphisms for $i<d$, and an epimorphism for $i=d$.

We are specially interested in the symplectic version of a particular case of Theorem 4.1 in this paper: whether the fundamental group of the space of regular values of a Lefschetz pencil generates the fundamental group of the complement of the dual variety. Our Theorem 4.2 is somewhat weaker than that, due to difficulties arising from the nature of the symplectic dual variety (see Question 6.1).

Let $M$ be a symplectic manifold of integer class and let $L \rightarrow M$ be the associated line bundle. For a compatible almost complex structure $J, \varepsilon>0$, and $k$ large enough, define

(1)

$$
\left.M_{\varepsilon, k, J}^{*}=\left\{s: M \rightarrow L^{\otimes k}: s \text { is } \varepsilon \text {-transverse, } s \text { has } C^{3} \text {-bounds } 1 \text { (for } J\right)\right\},
$$

with the natural $C^{3}$-topology. Observe that $M_{\varepsilon_{1}, k, j}^{*} \subset M_{\varepsilon_{2}, k, J}^{*}$, for $\varepsilon_{1} \geq \varepsilon_{2}$. Note first that the isotopy result of [3] shows that for $k$ large enough (how 
large depending on $\varepsilon$ ), the zero sets of the sections $s_{k} \in M_{\varepsilon, k, J}^{*}$ have isotopic zero sets $W_{k}=Z\left(s_{k}\right)$.

Now we aim to understand better the fundamental group of the spaces $M_{\varepsilon, k, J}^{*}$. We will study a suitably modified version of it. Let $\Gamma_{s}\left(L^{\otimes k}\right)$ denote the subspace of sections of $L^{\otimes k}$ whose zero locus is a smooth symplectic submanifold of $M$, and let $\mathbb{P} \Gamma_{s}\left(L^{\otimes k}\right)$ be its projectivization. Therefore the space $M_{\varepsilon, k, J}^{*}$ is an open subset of the space $\Gamma_{s}\left(L^{\otimes k}\right)$. We denote by $\rho_{\varepsilon, k, J}$ : $M_{\varepsilon, k, J}^{*} \rightarrow \mathbb{P} \Gamma_{s}\left(L^{\otimes k}\right)$ the natural map and set

$$
\pi_{\varepsilon, k, J}=\left(\rho_{\varepsilon, k, J}\right)_{*}\left(\pi_{1}\left(M_{\varepsilon, k, J}^{*}\right)\right) \subset \pi_{1}\left(\mathbb{P} \Gamma_{s}\left(L^{\otimes k}\right)\right) .
$$

Denote $\operatorname{Map}\left(W_{k}\right)=\operatorname{Diff}^{+}\left(W_{k}\right) / \operatorname{Diff}_{0}\left(W_{k}\right)$ the mapping class group of the fiber, i.e. its oriented diffeomorphisms modulo isotopies. As in the algebraic case there is a geometric monodromy map

$$
\mu: \pi_{1}\left(M_{\varepsilon, k, J}^{*}, *\right) \longrightarrow \operatorname{Map}\left(W_{k}\right)
$$

which factors through $\left(\rho_{\varepsilon, k, J}\right)_{*}$, thus defining a map $\pi_{\varepsilon, k, J} \rightarrow \operatorname{Map}\left(W_{k}\right)$.

The proposed way for studying the topology of $M_{\varepsilon, k, J}^{*}$ is by means of a Lefschetz hyperplane theorem that allows a finite dimensional reduction. The idea is to restrict ourselves to a linear subspace of finite dimension. More specifically, choose sufficiently generic sections $s_{k}^{0}, \ldots, s_{k}^{N}$ in $M_{\varepsilon, k, J}^{*}$ and let $V_{k, N}$ be their linear span. Then one may ask whether there is an isomorphism $\pi_{j}\left(V_{k, N} \cap M_{\varepsilon, k, J}^{*}\right) \cong \pi_{j}\left(M_{\varepsilon, k, J}^{*}\right)$ for $j$ below the middle dimension as in Theorem 4.1. In the case of the fundamental group we consider a symplectic Lefschetz pencil $\phi_{k}=s_{k}^{2} / s_{k}^{1}$, which defines a natural map:

$$
\begin{aligned}
\Psi_{k}: \mathbb{C P}^{1}-\left\{p_{1}, \ldots, p_{l}\right\} & \rightarrow \mathbb{P} \Gamma_{s}\left(L^{\otimes k}\right) \\
{\left[q_{1}, q_{2}\right] } & \mapsto\left[q_{1} s_{k}^{2}-q_{2} s_{k}^{1}\right]
\end{aligned}
$$

where $p_{1}, \ldots, p_{l} \in \mathbb{C P}^{1}$ are the images of the critical points.

Theorem 4.2. Let $\varepsilon>0$ be small enough. Then there exists $k_{0}$ such that for any $k \geq k_{0}$ the image of the geometric monodromy map

$$
\mu: \pi_{1}\left(M_{\varepsilon, k, J}^{*}\right) \rightarrow \operatorname{Map}\left(W_{k}\right)
$$

lies in the subgroup of $\operatorname{Map}\left(W_{k}\right)$ generated by the positive generalized Dehn twists of a single Donaldson's Lefschetz pencil $\phi_{k}: M-N_{k} \rightarrow \mathbb{C P}^{1}$.

Proof. First we need to introduce some definitions. Abusing notation we will denote by $L$ the pull-back of the bundle $L$ to the manifold $M \times S^{1}$. Then we can define $C^{3}$-bounds of a sequence of sections of $L^{\otimes k} \rightarrow M \times S^{1}$ just by requiring that the restriction to each fiber of $\pi: M \times S^{1} \rightarrow S^{1}$ satisfy these bounds. In the same way the section $s: M \times S^{1} \rightarrow L^{\otimes k}$ is $\varepsilon$-transverse to zero if the restriction to the fiber $\pi^{-1}(z)$ is $\varepsilon$-transverse for any $z \in S^{1}$. 
We fix, once for all, as a base point, a section $s_{k, 0}^{2} \in M_{\varepsilon, k, J}^{*}$, that exists by [9] for some small $\varepsilon>0$. Take an element $A \in \pi_{1}\left(M_{\varepsilon, k, J}^{*}, s_{k, 0}^{2}\right)$. Fix a representative $\alpha: S^{1} \rightarrow M_{\varepsilon, k, J}^{*}$, i.e., $[\alpha]=A$. The map $\alpha$ defines canonically a section $s_{k}^{2}$ of $L^{\otimes k} \rightarrow M \times S^{1}$ by the formula

$$
s_{k}^{2}\left(x, e^{2 \pi i t}\right)=\left(\alpha\left(e^{2 \pi i t}\right)\right)(x) .
$$

with the base point condition that $s_{k}^{2}(\cdot, 1)=s_{k, 0}^{2}$. It is clear that $s_{k}^{2}$ has $C^{3}$-bounds 1 and it is $\varepsilon$-transverse to zero. Now, choose another $\varepsilon$-transverse section $s_{k}^{1}$ of $L^{\otimes k} \rightarrow M$ such that $s_{k}^{1} \oplus s_{k, t}^{2}$ is a section of $L^{\otimes k} \oplus L^{\otimes k}$ with $C^{3}$-bounds 1 for all $t \in S^{1}$. Moreover we may also assume that $s_{k}^{1} \oplus s_{k, j}^{2}$ is already an $\varepsilon$-transverse Lefschetz pencil (for $\varepsilon$ small enough), for $j=0,1$. Denote again by $s_{k}^{1}$ the pull-back of the section to $M \times S^{1}$. Applying the $S^{1}$-parametric version of Propositions 2.7 and 2.8 we perturb $s_{k}^{1} \oplus s_{k}^{2}$ into $\sigma_{k}^{1} \oplus \sigma_{k}^{2}$, a new section that defines a $S^{1}$-family of $\varepsilon^{\prime}$-transverse Lefschetz pencils, for some $\varepsilon^{\prime}<\varepsilon$.

Note that in the process of Proposition 2.7 it is necessary to perturb only $s_{k, t}^{2}$. This is because the condition (ii) is already satisfied by $s_{k, t}^{1}=s_{k}^{1}$, the condition (iv) is obtained in [10] only perturbing $s_{k, t}^{2}$ and the proof of the condition (iii) can be changed using the alternative proof written in [3] that does not need to perturb $s_{k}^{1}$. So $\sigma_{k}^{1}=s_{k}^{1}$. Moreover, the perturbation in $s_{k, t}^{2}$ can be very small so that the sequence of sections $\sigma_{k, t}^{2}$ is isotopic to the previous one. Also we may assume that $\sigma_{k, j}^{2}=s_{k, j}^{2}$ for $j=0,1$, since $s_{k}^{1} \oplus \sigma_{k, j}^{2}$ are already Lefschetz pencils.

Now we want to compute the monodromy associated to the zero set of $\sigma_{k}^{2}$, which is the same as the one of $s_{k}^{2}$.

For each fiber $M \times\left\{e^{2 \pi i t}\right\}$ we know that the images of the critical points of the associated Lefschetz pencil $\phi_{k, t}$ are a finite number of disjoint points. For the whole family of pencils over $S^{1}$ the critical values describe a braid in $\mathbb{C P}^{1}$, so we can choose a continuous family of paths $\lambda_{t}:[0,1] \rightarrow \mathbb{C P}^{1}$ joining 0 with $\infty$, such that each $\lambda_{t}$ avoids the critical values of the pencil $\phi_{k, t}$.

Now, the disk bounded by $\sigma_{k, t}^{2}, \lambda_{0}, \lambda_{1}$ and the constant path $s_{k}^{1}$ defines a base-pointed homotopy between the loops $\sigma_{k, t}^{2}$ and $\lambda_{0}^{-1} \cdot \lambda_{1}$. The latter loop is contained in the regular values of the Lefschetz pencil $\phi_{k, 0}=\left[s_{k}^{1}, \sigma_{k, 0}^{2}\right]$, therefore the monodromy of the family of sections of $M$ over it is a product of direct generalized Dehn twists.

As $\varepsilon^{\prime}$ depends on $\varepsilon$, we may find $k_{\varepsilon}$ only dependent on $\varepsilon$ such that, for any $k \geq k_{\varepsilon}$, all $\varepsilon^{\prime}$-transverse pencils are isotopic. Furthermore the isotopy between two pencils $\left[s_{k}^{1}, s_{k, 0}^{2}\right]$ and $\left[\sigma_{k}^{1}, s_{k, 0}^{2}\right]$ of $M$ may be achieved by a family of pencils that keeps fixed the section $s_{k, 0}^{2}$. Thus the image of the geometric monodromy map $\mu$ is contained in the subgroup of $\operatorname{Map}\left(W_{k}\right)$ generated by a single pencil, which may be assumed to be $\varepsilon$-transverse. 


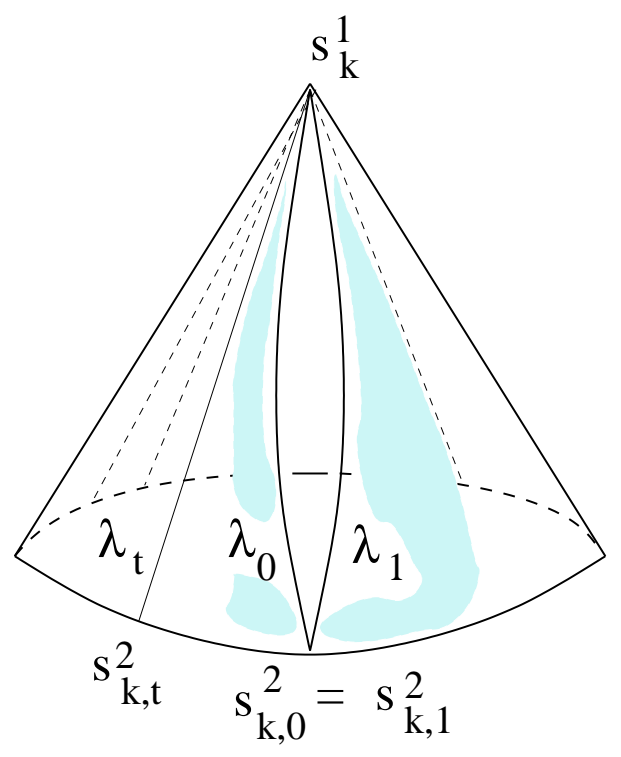

Figure 1. Homotopy between $\sigma_{k, t}^{2}$ and $\lambda_{0}^{-1} \cdot \lambda_{1}$ in $M_{\varepsilon, k, J}^{*}$.

\section{Nontriviality of the vanishing spheres}

Now we will prove Theorems 1.3 and 1.4. The first one is an immediate consequence of Theorem 4.2.

Proof of Theorem 1.3. Recalling the proof of Theorem 1.1, take two vanishing spheres $S_{0}, S_{1}$ in a fiber of a Donaldson Lefschetz pencil and join them by a path of Lagrangian spheres $S_{t}$ such that $S_{t} \subset W_{t}^{\prime}$, where $W_{t}^{\prime}$ is a family of fibers in a family of pencils $\phi_{k, t}$. This means that $W_{t}^{\prime}=Z\left(s_{k, t}\right)$, $s_{k, t} \in M_{\varepsilon, k, J}^{*}$. We can suppose that $s_{k, 0}=s_{k, 1}$ is the base point. Now $s_{k, t}$ can be homotoped, by Theorem 4.2, to a path inside a Lefschetz pencil whose zero set we denote by $W_{t}$. As usual, parallel transport along the path generates a family of symplectomorphisms $\phi_{t, s}: W_{t} \rightarrow W_{t+s}$. The symplectomorphism $\phi_{0,1}$ is generated by the composition of the generalized Dehn twists associated to the critical points of the pencil bounded by the path.

However $S_{0}$ is not, in general, preserved by $\phi_{0,1}$. Denote by $S_{t, s}=\phi_{t, s}\left(S_{t}\right)$. Then we have that the continuous family $S_{t, 1-t}$ provides a Lagrangian isotopy in $W_{0}=W_{1}$ between $S_{1}=S_{1,0}$ and $S_{0,1}$. This shows that the spheres $S_{0}$ and $S_{1}$ are conjugated by $\phi_{0,1}$, up to Lagrangian isotopy.

Remark 5.1. The spheres are conjugated only up to Lagrangian isotopy. We can also claim that they are conjugated up to Hamiltonian isotopy because the obstruction of a Lagrangian isotopy to be lifted to a Hamiltonian isotopy lies in the family of groups $H^{1}\left(L_{t}\right)$ (cf. Exercise 6.1.A in [15]). This 
is an infinitesimal condition to be fulfilled for each member of the family. In our case this cohomology groups vanish in the case of dimension strictly bigger than 4 . In dimension 4 , we can check that the spheres at the starting and end points of the family are exact (using the local models close to critical points) and this is enough to get a Hamiltonian isotopy (recall that in dimension 4 the fibers are Riemann surfaces).

Theorem 1.3 admits an extension from the symplectomorphism group of the fiber to that of the total space.

Theorem 5.2. For a Donaldson's pencil on $M$ with $k$ large enough, fix a generic fiber $W$. If $S_{0}, S_{1} \subset W$ are Lagrangian spheres that appear as vanishing cycles of the pencil then there exists a symplectomorphism $\Phi$ : $M \rightarrow M$ such that $\Phi(W)=W$ and $\Phi\left(S_{0}\right)$ equals $S_{1}$ up to Lagrangian isotopy in the fiber $W$.

Proof. It follows from Proposition 3.1 that there exists a loop of approximately holomorphic sections $s_{k, t}$ such that for $t=0,1$ the zero set $Z\left(s_{k, t}\right)=$ $W$, and there is a family of Lagrangian spheres $S_{t}$ in $W_{t}=Z\left(s_{k, t}\right)$ connecting the two given vanishing spheres.

Now by Proposition 4 in [3], there is a continuous family of symplectomorphisms $\phi_{t}: M \rightarrow M$ such that $\phi_{t}\left(W_{0}\right)=W_{t}$. Again, we cannot assume that $S_{t}$ is preserved by the family. However, if we construct $S_{t, s}=\phi_{s}\left(S_{t}\right) \subset W_{t+s}$, we have that the family $S_{t, 1-t}$ defines a Lagrangian isotopy in $W_{1}=W_{0}$ between $S_{1}$ and $S_{0,1}=\phi_{1}\left(S_{0}\right)$. So, we have shown, up to Lagrangian isotopy in the fiber, that $\phi_{1}$ is the required symplectic map.

Proof of Theorem 1.4. The proof of Theorem 1.4 works looking for a contradiction. Theorem 1.3 implies that all the vanishing spheres are either homologically trivial or homologically non-trivial. Let us suppose that they are homologically trivial.

Let $\phi_{k}: M-N_{k} \rightarrow \mathbb{C P}^{1}$ be the Lefschetz pencil obtained from Theorem 2.6 for $k$ large, and denote by $F_{k}$ a regular fiber. We blow-up the manifold $M$ along $N_{k}$ to obtain a fibration $\tilde{\phi}_{k}: \tilde{M}_{k} \rightarrow \mathbb{C P}^{1}$.

Now let us compute the (growth of the) Betti numbers of $\tilde{M}_{k}$. First, by [3, Proposition 5], $\chi\left(F_{k}\right)=(-1)^{n-1} \operatorname{vol}(M) k^{n}+O\left(k^{n-1}\right)$, where $\operatorname{vol}(M)=$ $\int_{M}\left(\frac{\omega}{2 \pi}\right)^{n}$. Now the Lefschetz hyperplane theorem [3] implies that $b_{i}\left(F_{k}\right)=$ $b_{i}(M)$, which is uniformly bounded for $i<n-1$ (i.e., independently of $k$ ). By Poincaré duality, $b_{2 n-2-i}\left(F_{k}\right)$ is also uniformly bounded. Therefore

$$
b_{n-1}\left(F_{k}\right)=\operatorname{vol}(M) k^{n}+O\left(k^{n-1}\right) .
$$

A handlebody decomposition of $\tilde{M}_{k}$ is obtained as follows: take a tubular neighbourhood $\nu\left(F_{k}\right)$ of a smooth fiber $F_{k}$ of $\tilde{\phi}_{k}$, and attach $n$-handles along $(n-1)$-spheres embedded in the boundary $\partial \nu\left(F_{k}\right)=F_{k} \times S^{1}$ as the vanishing cycles at different fibers. Since all of these spheres are homologically trivial, 
the homology group $H_{n-1}$ does not change. So $H_{n-1}\left(\tilde{M}_{k}-F_{k} \times D^{2}\right) \cong$ $H_{n-1}\left(F_{k}\right)$. Finally, we have to attach $F_{k} \times D^{2}$ to get $\tilde{M}_{k}$. We have a Mayer-Vietoris exact sequence

$$
\begin{aligned}
H_{n-1}\left(F_{k} \times S^{1}\right) & \rightarrow H_{n-1}\left(\tilde{M}_{k}-F_{k} \times D^{2}\right) \oplus H_{n-1}\left(F_{k} \times D^{2}\right) \rightarrow \\
& \rightarrow H_{n-1}\left(\tilde{M}_{k}\right) \rightarrow H_{n-2}\left(F_{k} \times S^{1}\right)
\end{aligned}
$$

Using that $b_{n-2}\left(F_{k} \times S^{1}\right)$ is uniformly bounded, that $H_{n-1}\left(F_{k} \times S^{1}\right) \rightarrow$ $H_{n-1}\left(F_{k} \times D^{2}\right)$ is surjective with kernel uniformly bounded and (4), we get

$$
b_{n-1}\left(\tilde{M}_{k}\right)=\operatorname{vol}(M) k^{n}+O\left(k^{n-1}\right) .
$$

Analogously to the computation of $F_{k}$, and using that

$$
\chi\left(N_{k}\right)=(-1)^{n-2}(n-1) \operatorname{vol}(M) k^{n}+O\left(k^{n-1}\right),
$$

we get that all the Betti numbers of $N_{k}$ are bounded except for $b_{n-2}\left(N_{k}\right)=$ $(n-1) \operatorname{vol}(M) k^{n}+O\left(k^{n-1}\right)$. In the blow up $\tilde{M}_{k}$, the exceptional divisor $\tilde{N}_{k}$ is a fibration over $N_{k}$ by $\mathbb{C P}^{1}$. There is a spectral sequence with $E_{2}$-term $H_{*}\left(N_{k}\right) \otimes H_{*}\left(S^{2}\right)$ and abutting to $H_{*}\left(\tilde{N}_{k}\right)$. This gives that all $b_{i}\left(\tilde{N}_{k}\right)$ are bounded, for $i \neq n-2, n$, and that $b_{n-2}\left(\tilde{N}_{k}\right)=b_{n}\left(\tilde{N}_{k}\right)=(n-1) \operatorname{vol}(M) k^{n}+$ $O\left(k^{n-1}\right)$.

Consider the following pieces of long exact sequences in homology,

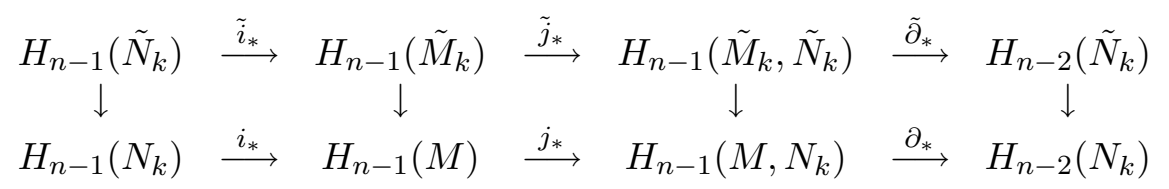

The kernel of $\tilde{i}_{*}$ is uniformly bounded, since $b_{n-1}\left(\tilde{N}_{k}\right)$ is so. Also $\operatorname{im} \tilde{j}_{*}=$ $\operatorname{ker} \tilde{\partial}_{*} \subset \operatorname{ker} \partial_{*}=\operatorname{im} j_{*}$ is uniformly bounded. Therefore, $b_{n-1}\left(\tilde{M}_{k}\right)$ is uniformly bounded. This is a contradiction with (5).

\section{Towards a symplectic invariant}

In the case of complex projective manifolds, the topology of the space of smooth sections of the ample bundle $L \rightarrow M$ is governed by the hyperplane section theorem of Hamm-Lê (Theorem 4.1), which in particular shows how the monodromy of generic families of dimension 1 or 2 of hyperplane sections capture the geometric monodromy of all families of sections.

In order to extend these properties from the complex algebraic to the symplectic case and define a new set of symplectic invariants, the next natural step is to prove that the groups $\pi_{\varepsilon, k, J}$ defined in (2), are independent of $J$ and of $\varepsilon$ for $k$ large enough. The authors have not been able to prove this, thus can only pose the following

Question 6.1 (symplectic Hamm-Lê hyperplane sections). Does the morphism $\pi_{l}\left(\mathbb{C P}^{j} \cap \mathbb{P} \Gamma_{s}\left(L^{\otimes k}\right)\right) \rightarrow \pi_{l}\left(\mathbb{P} \Gamma_{s}\left(L^{\otimes k}\right)\right)$ induce an isomorphism $\pi_{l}\left(\mathbb{C} \mathbb{P}^{j} \cap\right.$ 
BEHAVIOR OF ASYMPTOTICALLY HOLOMORPHIC LEFSCHETZ PENCILS 391

$\left.\mathbb{P} \Gamma_{s}\left(L^{\otimes k}\right)\right) \rightarrow\left(\rho_{\varepsilon, k, J}\right)_{*} \pi_{l}\left(M_{\varepsilon, k, J}^{*}\right)$ for $l<j$ and an epimorphism for $l=j$, for a $(j+1)$-dimensional "generic" family of Donaldson's $\varepsilon$-transverse sections?

An affirmative answer would yield a family of invariants, the $\varepsilon$-transverse homotopy groups of the complement $M_{\varepsilon, k, J}^{*}$ of the dual variety, which would be an extension of the Auroux and Katzarkov invariants of [5]. It must be warned that the only evidence available for an affirmative answer is the case of complex algebraic manifolds, and the authors have been able to make only a modest extension to the general symplectic case:

Corollary 6.2. For $\varepsilon>0$ small and $k$ large enough, we have

$$
\left(\rho_{\varepsilon, k, J}\right)_{*}\left(H_{1}\left(M_{\varepsilon, k, J}^{*}\right)\right)=\mathbb{Z} / m \mathbb{Z} \subset H_{1}\left(\mathbb{P} \Gamma_{s}\left(L^{\otimes k}\right)\right),
$$

where $m$ is a divisor of the number $n_{k}$ of singular fibers in the Lefschetz pencil $\phi_{k}: M-N_{k} \rightarrow \mathbb{C P}^{1}$.

Proof. By Theorem 4.2 the image of $H_{1}\left(M_{\varepsilon, k, J}^{*}\right)$ is generated by some compositions of monodromies around critical points of a single Donaldson's Lefschetz pencil. But the proof of Proposition 3.1 shows that all the loops around different critical points are freely homotopic. Therefore this abelian group is cyclic, generated by the 1-cycle $\gamma$ bounding a small disk $D$ around a critical value of the pencil.

The inclusion of the regular values of any Donaldson's Lefschetz pencil in $\mathbb{P} \Gamma_{s}\left(L^{\otimes k}\right)$ induces a homology $n_{k} \gamma \sim 0$.

The "natural" value for $m$ above should be the number $n_{k}$.

\section{References}

[1] V.I. Arnold, A. Varchenko and S. Goussein-Zadé, Singularités des applications differentiables, Mir, 1986.

[2] M. Audin, F. Lalonde and L. Polterovich, Symplectic rigidity: Lagrangian submanifolds, in 'Holomorphic curves in symplectic geometry' (M. Audin, J. Lafontaine, eds.), PM, 117, Birkhäuser, 1994.

[3] D. Auroux, Asymptotically holomorphic families of symplectic submanifolds, Geom. Funct. Anal. 7 (1997), 971-995.

[4] _ Symplectic 4-manifolds as branched coverings of $\mathbb{C P}^{2}$, Invent. Math. 139 (2000), 551-602.

[5] D. Auroux and L. Katzarkov, Branched coverings of $\mathbb{C P}^{2}$ and invariants of symplectic 4-manifolds, Invent. Math. 142 (2000), 631-673.

[6] D. Auroux, S. Donaldson, L. Katzarkov and M. Yotov, Fundamental groups of complements of plane curves and symplectic invariants, math.GT/0203183.

[7] D. Auroux, V. Muñoz and F. Presas, Lagrangian submanifolds and Lefschetz pencils, math.SG/0407126.

[8] V. Braungardt and D. Kotschick, Clustering of critical points in Lefschetz fibrations and the symplectic Szpiro inequality, math.SG/0204153. 
[9] S.K. Donaldson, Symplectic submanifolds and almost-complex geometry, J. Differential Geom. 44 (1996), 666-705.

[10] _ Lefschetz pencils on symplectic manifolds, J. Differential Geom. 53 (1999), 205-236.

[11] M. Goresky and R. MacPherson, Stratified Morse theory, Ergebnisse der Mathematik und ihrer Grenzgebiete (3), 14, Springer-Verlag, Berlin, 1988.

[12] A. Kas, On the handlebody decomposition associated to a Lefschetz fibration, Pacific J. Math. 89 (1980), 89-104.

[13] D. McDuff and D. Salamon, Introduction to symplectic topology, Oxford Math. Monographs, 2nd ed., 1998.

[14] V. Muñoz, F. Presas and I. Sols, Almost holomorphic embeddings in grassmanians with applications to singular symplectic submanifolds, J. Reine Angew. Math. 547 (2002), 149-189.

[15] L. Polterovich, The geometry of the group of symplectic diffeomorphisms, Birkhäuser, Lectures is Mathematics, Basel, 2001.

[16] I. Smith, Lefschetz pencils and divisors in moduli space, Geom. and Topology 5 (2001), 579-608.

[17] I. Smith, R.P. Thomas and S.-T. Yau, Symplectic conifold transitions, J. Differential Geom. 62 (2002), 209-242.

[18] Seminaire de Geometrie Algebrique 7-2, Lecture Notes Math., 340, Springer-Verlag.

Departament de Matemàtica Aplicada I

Universitat Politècnica de Catalunya

08028 BARcElona, Spain

E-mail address: jaume.amoros@upc.es

Departamento de Matemáticas

Universidad AutónOma DE MADRID

28049 MADRID, SPAIN

E-mail address: vicente.munoz@uam.es

Departamento de Matemáticas

Universidad AUtónoma de MADRID

28049 MADRID, SPAIN

E-mail address: francisco.presas@uam.es

First author was partially supported by CICYT BFM2003-06001. Second author was partially supported by CICYT BFM2000-0024. Third author was partially supported by CICYT BFM2000-0024 and by Fundación Pedro Barrié de la Maza. All authors were partially supported by The European Contract Human Potential Programme, Research Training Network HPRN-CT-2000-00101. 